\title{
Coronary vasospasm induced by painful compression of the brachial artery following coronary angiography
}

\author{
A. O. Kraaijeveld • D. J. van der Heijden • A. Sieders • \\ C. J. H. J. Kirchhof
}

Published online: 19 April 2013

(C) The Author(s) 2013. This article is published with open access at Springerlink.com

\begin{abstract}
We report the occurrence of coronary vasospasm following forceful compression of the brachial artery after coronary angiography.
\end{abstract}

Keywords Coronary angiography $\cdot$ Vasospasm $\cdot$ Pain · Autonomic nervous system

\section{Introduction}

The autonomic nervous system has been shown to play an important role in the aetiology of coronary vasospasm [1]. Both parasympathetic and sympathetic triggers may provoke coronary vasospasm [2, 3]. Nociceptor stimulation affects autonomic balance significantly. However, there are no case reports describing the occurrence of local pain as a trigger for coronary spasm. We present a case of painful brachial artery compression resulting in coronary vasospasm.

\section{Case report}

A 69-year-old female patient was admitted to our emergency department with complaints of recurrent short-lasting chest pain during the night in the previous 2 weeks. The chest pain typically occurred every night around 3 A.M., lasting for approximately $3 \mathrm{~min}$. Her medical history mentioned hypertension, and a left-sided T1N0M0 breast carcinoma curatively treated with surgery and radiotherapy 14 years

\footnotetext{
A. O. Kraaijeveld $(\bowtie)$

Department of Cardiology, C5-P, Leiden University Medical Center, PO Box 9600, 2300 RC Leiden, the Netherlands e-mail: a.o.kraaijeveld@lumc.nl

D. J. van der Heijden • A. Sieders • C. J. H. J. Kirchhof Department of Cardiology, Rijnland Hospital, Leiderdorp, the Netherlands
}

earlier. Analysis for coronary artery disease at the age of 53 showed no abnormalities. Her medication included aspirin, thiazide diuretics and a low maintenance dose of nitrates.

On the morning of admission, she experienced the same chest pain with radiation to her neck and complaints of sweating. Sublingual nitrates administered at the emergency department relieved her pain to some extent. There were no abnormalities found during physical examination.

The electrocardiogram on admission showed sinus rhythm, normal axis and negative $\mathrm{T}$ waves in the anterolateral leads (Fig. 1a). Since troponin-T levels were slightly elevated $(0.11 \mu \mathrm{g} / \mathrm{L})$ she was treated for a non-ST-elevation myocardial infarction (NSTEMI) with clopidogrel, low-molecular-weight heparins, statins, beta-blockers as well as intravenous nitrates in addition to her previous medication. During hospitalisation, the maximum troponin $\mathrm{T}$ level was $0.34 \mu \mathrm{g} / \mathrm{L}$, creatine kinase 335 U/L. Serial electrocardiograms during chest pain showed repolarisation abnormalities indicative for myocardial ischaemia in the anterior and inferior wall leads as well as QTc prolongation of 490 milliseconds, suggesting a proximal left coronary artery and possibly right coronary artery stenosis as well (Fig. 1b). Diagnostic coronary angiography was performed within $24 \mathrm{~h}$, the next day. Modified Seldinger procedures in both femoral and right radial arteries were complicated by difficult vascular access. Eventually, the right brachial artery was accessed. Coronary angiography revealed a markedly large left anterior descending artery (LAD), together with a non-dominant right coronary artery. Minor wall abnormalities in both the right and the left coronary artery were found, with no signs of significant coronary stenosis (Fig. 2). After removing the arterial sheath, haemostasis was accomplished by manual compression of the brachial artery, followed by a pressure bandage for $4 \mathrm{~h}$.

Thirty minutes after removal of the arterial sheath, the lower right arm turned white and became cold and painful, due to arterial occlusion. The pressure bandage was carefully removed. Shortly thereafter, she again experienced chest pain. 
a

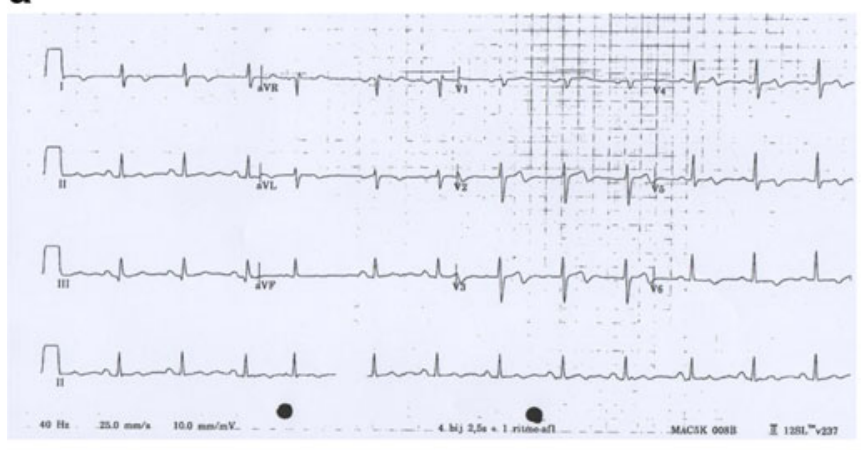

C

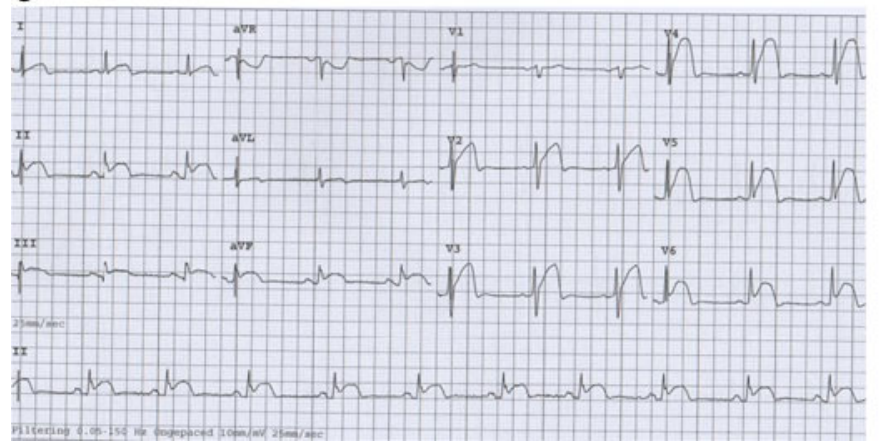

b

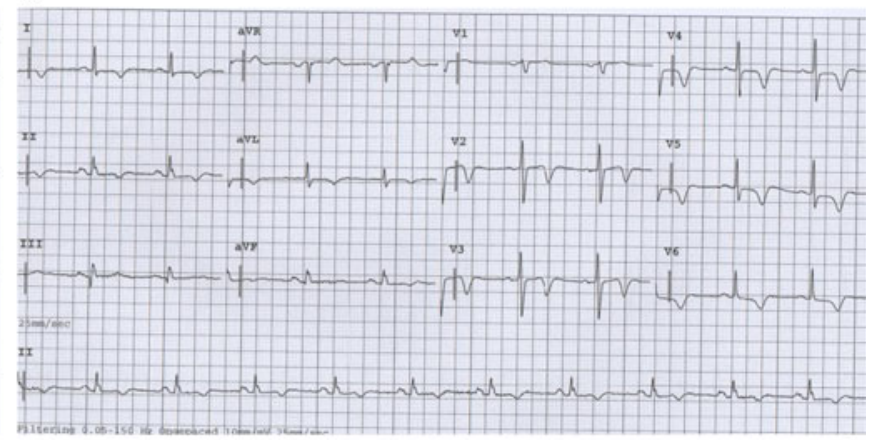

Fig. 1 Serial recorded electrocardiograms during hospitalisation. a At the emergency department, during admission. b After admission, no symptoms. c During chest pain, after experiencing severe pain in the right arm. ST elevation is present in the inferior and anterior wall leads

The electrocardiogram showed sinus bradycardia with marked ST elevation in the precordial leads as well as in the inferior wall leads (Fig. 1c). Considering the anatomy of the LAD running over the apex towards the inferior wall, this pattern was consistent with a proximal LAD occlusion, or with a combined LAD and right coronary artery (RCA) spasm. This leaves coronary vasospasm as the only possible explanation of her symptoms. No arrhythmias were seen. Intravenous nitroglycerin was immediately administered resolving her

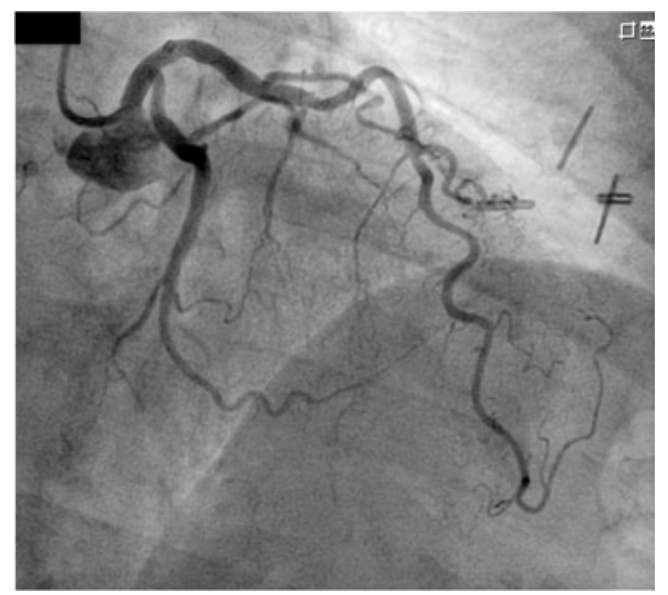

Fig. 2 RAO $30^{\circ}$ projection of the left anterior descending (LAD) and the circumflex arteries, showing a large LAD which runs over the tip of the cardiac apex. The proximal LAD contains wall irregularities without significant coronary stenoses symptoms, and the electrocardiogram showed marked Twave inversion as was seen previously (Fig. 1b). She was treated with a calcium antagonist accordingly and was free of symptoms during outpatient follow-up.

\section{Discussion}

In conclusion, coronary vasospasm followed within minutes of a painful stimulus. To our knowledge, this is the first report describing such a case. Although we do not have direct angiographic proof using intracoronary provocation testing, this is a typical case. In retrospect, her symptoms of chest pain during the night are typical for coronary vasospasm and were probably caused by enhanced parasympathetic activity during sleep. Various studies have demonstrated a relationship between autonomic imbalance and coronary vasospasm [2-4].

After coronary angiography, the painful compression of her left arm induced vagal activation, evidenced by sinus bradycardia. As a compensatory mechanism the sympathetic nervous reflex was enhanced, leading to coronary vasospasm by affecting vasomotor tone. This concept has been described previously $[2,3]$, and was also demonstrated in a patient with coronary vasospasm $4 \mathrm{~min}$ after carotid sinus massage [5]. In our case, the same sequence of events occurred within minutes. We did not verify the occurrence of her symptoms with a similar painful stimulus since she responded well to medical treatment. 
The presence of atherosclerosis in the proximal LAD could eventually serve as the substrate for coronary vasospasm, although a combination of LAD and RCA spasm cannot be excluded. Endothelial dysfunction enhances vascular smooth muscle cell contractility by decreased nitric oxide synthase [1, 6]. Statin treatment is advised, although no clinical trial exists on its effect in coronary vasospasm. Treatment of variant angina in most cases suffices with calcium antagonists and/or nitrates. Prognosis is good, as recently confirmed by the CASPAR study [7], although persistent angina in some cases remains a challenging problem.

Disclosures The patient has given written informed consent.

\section{Conflict of interests None declared.}

Open Access This article is distributed under the terms of the Creative Commons Attribution License which permits any use, distribution, and reproduction in any medium, provided the original author(s) and the source are credited.

\section{References}

1. Stern S, Bayes de Luna A. Coronary artery spasm: a 2009 update. Circulation. 2009;119:2531-4.

2. Yasue H, Touyama M, Shimamoto $M$, et al. Role of autonomic nervous system in the pathogenesis of prinzmetal's variant form of angina. Circulation. 1974;50:534-9.

3. Lanza GA, Pedrotti P, Pasceri V, et al. Autonomic changes associated with spontaneous coronary spasm in patients with variant angina. J Am Coll Cardiol. 1996;28:1249-56.

4. Pozzati A, Pancaldi LG, Di Pasquale G, et al. Transient sympathovagal imbalance triggers "ischemic" sudden death in patients undergoing electrocardiographic holter monitoring. J Am Coll Cardiol. 1996; 27:847-52.

5. Nishizaki M, Yamawake N, Arita M. Coronary artery spasm induced by carotid sinus massage. Heart. 2000;84:E2.

6. Turgut O, Tandogan I, Sarikaya S. Spontaneous resolution of midsegment coronary vasospasm previously unresponsive to intracoronary nitroglycerin. Neth Heart J. 2011;19:355-6.

7. Ong P, Athanasiadis A, Borgulya G, et al. 3-year follow-up of patients with coronary artery spasm as cause of acute coronary syndrome: the caspar (coronary artery spasm in patients with acute coronary syndrome) study follow-up. J Am Coll Cardiol. 2011; $57: 147-52$ 